\title{
液态金属上“赏”石墨烯舞动
}

汪璐阳, 曾梦琪, 付䂞*

武汉大学化学与分子科学学院, 武汉 430072

*联系人, E-mail: leifu@whu.edu.cn

\section{Observing graphene dancing on liquid metal}

\author{
Luyang Wang, Mengqi Zeng \& Lei Fu ${ }^{*}$ \\ College of Chemistry and Molecular Sciences, Wuhan University, Wuhan 430072, China \\ * Corresponding author, E-mail: leifu@whu.edu.cn
}

doi: 10.1360/TB-2021-0759

在大自然中, 我们常会看到落叶随水流漂动之景, 其在 水表面上自由漂浮与水的流动性是密不可分的. 液体正是由 于这种独特的性质, 为我们呈现了别样的动态之美. 在二维 材料世界中, 液态金属充当着兼具催化性能和流动性的生长 基底的角色，为二维材料的可控合成与自组装注人了新的 活力 ${ }^{[1]}$.

\section{1 以液态之柔, 托舞者之形}

大尺寸、无缺陷石墨烯的可控合成是推动其走向实际 应用的关键. 在众多的合成方法中，基于金属催化剂的化学 气相沉积法脱颖而出, 它被认为是制备高质量石墨烯的最有 效途径之一. 在石墨烯的合成过程中，传统的固态金属催化 剂由于自身存在的较大的表面粗粘度、丰富的晶界和多样 缺陷，会导致不均匀地成核，可控合成高质量石墨烯面临着 多重挑战. 而当金属变为液态时，这些不均匀的表面结构会 消失，金属展现出光滑且可流变的一面，正是由于这种“刚” 至“柔”的转变, 为石墨烯的生长带来了更多的可能性, 一些 神奇的现象在液态表面孕育而生. 在液态金属表面生长的石 墨烯如舞者般, 为我们呈现出精彩纷呈的画面: 在液态金属 上，研究者观察到了具有更加丰富形貌的石墨烯单晶 ${ }^{[2]}$, 也 注意到圆形的石墨烯等二维材料单晶可以发生平滑拼接 ${ }^{[3 \sim 5]}$, 还发现石墨烯单晶像施了魔法般可以进行自组装，有序排列 成阵列 ${ }^{[6]}$.

\section{2 借原位之力, 赏舞者之姿}

神奇现象的神秘面纱尚未被揭开, 液态金属上石墨烯的 生长过程仍是一个“黑匣子”, 研究者只看到了结果, 对于这 些现象背后的成因只能依赖于非原位实验和理论计算来进
行推测, 尚不能全面理解其中生长和组装的全过程. 如果我 们能直观地看到整个动态过程, 这将会有力推动对石墨烯在 液态金属表面生长和运动行为的认知. 原位技术则成为了解 决该问题的突破口. 本研究团队结合前期在液态金属上生长 石墨烯的经验，利用原位成像技术揭示了液态金属表面石墨 烯的生长和运动机制, 相关成果于 2021 年7月 8 日在线发表在 Advanced Science ${ }^{[7]}$.

本团队搭建了一套原位装置为原位可视化石墨烯在液 态铜表面的生长过程提供了平台(图1). 其中, 具有观察窗口 的高温原位反应台可以保证在反应可视化的同时提供足够 的热量使金属熔化; 热辐射模式观察装置能够基于石墨烯与 液态铜在高温下热辐射的差异将二者区分开，保证了对反应 过程的实时有效追踪. 同时, 通过原位平台的实时反馈, 我们 可以监测不同生长参数下石墨烯的成核和生长信息.

借助原位平台, 液态铜表面石墨烯的生长、运动及自组 装行为被全面地展现出来，本团队见证了石墨烯犹如“舞者” 般在液态金属“舞台”上的精彩瞬间．石墨烯舞者们在液态金 属舞台上平移、旋转、形成队形, 它们灵活地舞动着, 这为 石墨烯在液态金属表面的运动行为提供了直接的证据. 液态 铜的流动性是石墨烯单晶能在其发生平移和旋转的前提条 件, 铜液面的微扰动会引起石墨烯运动路径的改变.

随后，我们对石墨烯单晶整齐队形背后的成因展开了探 索，原位成像技术能够实时捕捉石墨烯单晶在液态铜表面自 组装的动态过程. 在生长和组装过程中, 组装过程是伴随生 长发生的, 基于最小表面能原则, 石墨烯单晶在生长的过程 中自发地在液态铜表面运动并趋向于均匀分布; 随着生长过 程的进行，石墨烯单晶之间距离减小，单晶间存在相互作用 迫使相邻石墨烯单晶发生移动和旋转并最终对准形成有序 


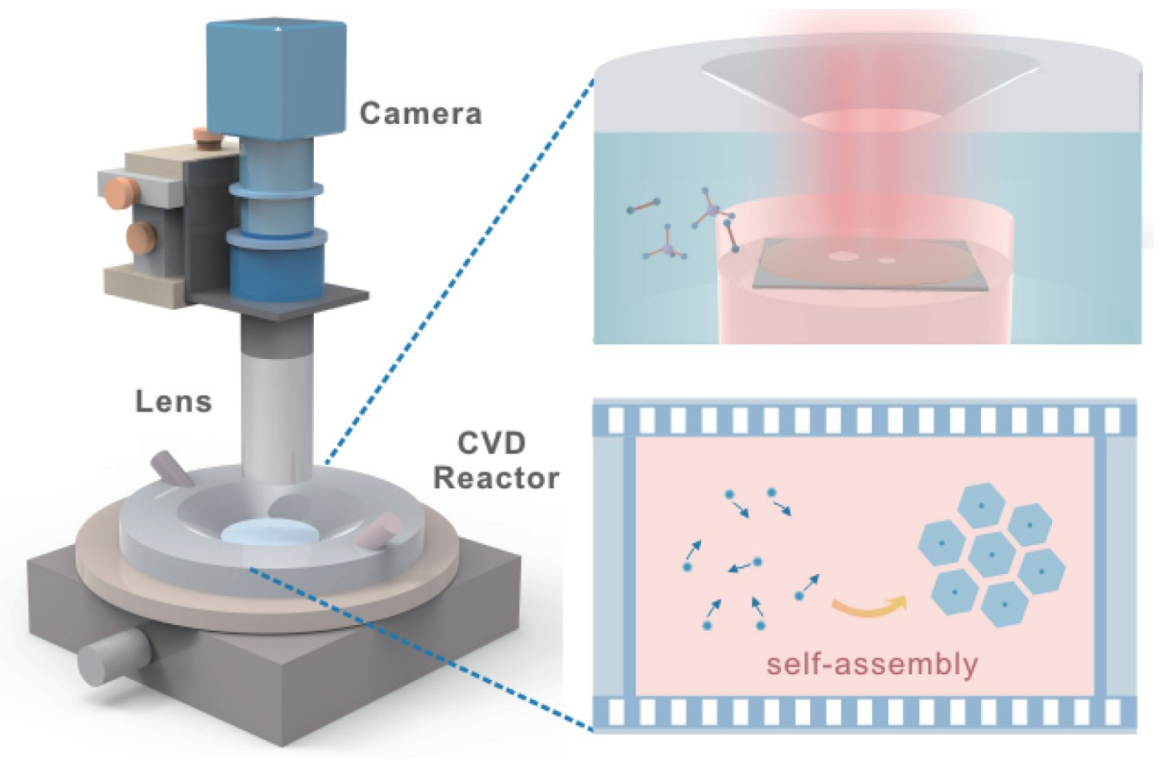

图 1 (网络版彩色)原位观测液态金属表面石墨烯生长和组装的示意图

Figure 1 (Color online) Schematic diagram of in-situ observing the growth and assembly of graphene on liquid metal surface

阵列, 此时的石墨烯单晶组装体趋于稳定; 如果进一步延长 生长时间，相邻石墨烯单晶间的间隙会消失，石墨烯单晶将 融合在一起. 在此基础上，通过调节石墨烯生长时间和前驱 体甲烷的浓度，实时监测阵列的组装情况，可以灵活调控石 墨烯组装体的周期性.

\section{3 结语}

液态金属为石墨烯的可控生长和组装带来了新的契机,
本团队利用原位成像技术可视化了液态金属表面石墨烯的 生长和运动的动态过程, 阐释了石墨烯在液态铜表面自组装 的机制，有助于进一步指导二维原子晶体的精准组装并促进 其在集成领域的应用，我们相信石墨烯舞者会在液态金属的 舞台上绽放更加夺目的光彩. 此外，原位技术的独特潜力值 得被进一步发掘, 通过多技术联用的手段, 从多个尺度更加 全面地认识反应过程, 从而促进对化学气相沉积过程中二维 材料的生长机制的理解.

\section{推荐阅读文献}

1 Zeng M, Fu L. Controllable fabrication of graphene and related two-dimensional materials on liquid metals via chemical vapor deposition. Acc Chem Res, 2018, 51: 2839-2847

2 Liu J X, Fu L. Controllable growth of graphene on liquid surfaces. Adv Mater, 2019, 31: 1800690

3 Tan L, Han J, Mendes R G, et al. Self-aligned single-crystalline hexagonal boron nitride arrays: Toward higher integrated electronic devices. Adv Electron Mater, 2015, 1: 1500223

4 Zeng M, Tan L, Wang L, et al. Isotropic growth of graphene toward smoothing stitching. ACS Nano, 2016, 10: 7189-7196

5 Lee J S, Choi S H, Yun S J, et al. Wafer-scale single-crystal hexagonal boron nitride film via self-collimated grain formation. Science, 2018, 362: $817-821$

6 Zeng M, Wang L, Liu J, et al. Self-assembly of graphene single crystals with uniform size and orientation: The first 2D super-ordered structure. J Am Chem Soc, 2016, 138: 7812-7815

7 Wang L, Ding Y, Wang X, et al. In situ investigation of the motion behavior of graphene on liquid copper. Adv Sci, 2021, 8: 2100334 